\title{
EFFECTS OF AMRINONE, A PHOSPHODIESTERASE INHIBITOR, ON RIGHT VENTRICULAR/ARTERIAL COUPLING IMMEDIATELY AFTER CARDIAC OPERATIONS
}

Yoshie Ochiai, MD

Shigeki Morita, MD

Yoshihisa Tanoue, MD

Yoshito Kawachi, MD

Ryuji Tominaga, MD

Hisataka Yasui, MD
Objective: Amrinone, a selective phosphodiesterase III inhibitor, is reported to have a potent inotropic effect on the left ventricle, but the effects of this drug on right ventricular contractility in the clinical setting are unknown. The concept of ventricular/arterial coupling was applied to investigate the effects of amrinone on right ventricular contractility and afterload with transesophageal echocardiography. Methods and results: The study was performed in the intensive care unit with 11 patients who had undergone cardiac operations. Right ventricular cross-sectional area was measured with transesophageal echocardiography equipped with a capability of automated border detection as a surrogate for right ventricular volume. Multiple pressure-area loops were obtained by reducing preload to measure end-systolic elastance and effective arterial elastance. External work and pressure-volume area were also measured to calculate the efficiency of energy transfer from pressure-volume area to external work. Nitroprusside (0.3 to $\left.0.5 \mu \mathrm{g} \cdot \mathrm{kg}^{-1} \cdot \mathrm{min}^{-1}\right)$ and amrinone $\left(1 \mathrm{mg} \cdot \mathrm{kg}^{-1}\right.$ intravenously followed by $10 \mu \mathrm{g} \cdot \mathrm{kg}^{-1} \cdot \mathrm{min}^{-1}$ ) were administered. With nitroprusside infusion, end-systolic elastance and effective arterial elastance remained unchanged (end-systolic elastance, $4.73 \pm 2.18 \mathrm{~mm} \mathrm{Hg} / \mathrm{cm}^{2}$ to $4.65 \pm 2.09 \mathrm{~mm}$ $\mathrm{Hg} / \mathrm{cm}^{2}$; effective arterial elastance, $8.05 \pm 3.84 \mathrm{~mm} \mathrm{Hg} / \mathrm{cm}^{2}$ to $7.70 \pm 3.64$ $\mathrm{mm} \mathrm{Hg} / \mathrm{cm}^{2}$ ). In contrast, amrinone reduced afterload (effective arterial elastance, $8.82 \pm 3.99 \mathrm{~mm} \mathrm{Hg} / \mathrm{cm}^{2}$ to $7.05 \pm 4.01 \mathrm{~mm} \mathrm{Hg} / \mathrm{cm}^{2}, p=0.004$ ) and enhanced contractility (end-systolic elastance, $4.47 \pm 1.79 \mathrm{~mm} \mathrm{Hg} / \mathrm{cm}^{2}$ to $\left.6.56 \pm 2.22 \mathrm{~mm} \mathrm{Hg} / \mathrm{cm}^{2}, p=0.007\right)$. Consequently, amrinone decreased the ventricular/arterial coupling ratio (effective arterial elastance/endsystolic elastance, $2.40 \pm 1.45$ to $1.16 \pm 0.63, p=0.009)$ and improved the efficiency of energy transfer (external work/pressure-volume area, $0.44 \pm$ 0.15 to $0.54 \pm 0.15, p=0.013)$. Conclusions: Right ventricular pressure-area relations obtained with transesophageal echocardiography could successfully separate the simultaneous change in right ventricular systolic mechanics and afterload caused by amrinone. Amrinone caused enhancement of right ventricular contractility and afterload reduction. ( $J$ Thorac Cardiovasc Surg 1998;116:139-47)
$A^{n}$ mong clinically applicable inotropic agents, amrinone, a selective phosphodiesterase III inhibitor, has been shown to have a positive inotropic effect with remarkable vasodilatory effect., ${ }^{1,2}$ Right ventricular afterload may be acutely elevated after

From the Department of Cardiovascular Surgery, Faculty of Medicine, Kyushu University, Fukuoka, Japan.

Received for publication Sept. 7, 1997; revisions requested Oct. 31, 1997; revisions received Dec. 16, 1997; accepted for publication Jan. 30, 1998.

Presented in part at the Sixty-ninth Scientific Sessions of the American Heart Association, New Orleans, La., November termination of cardiopulmonary bypass because of the inflammatory reaction associated with extracorporeal circulation that causes pulmonary hypertension and lung injury. ${ }^{3}$ In patients after cardiac operations, amrinone reduces pulmonary artery

10-13, 1996, and published in abstract form (Circulation 1996;94(Suppl):I379).

Address for reprints: Shigeki Morita, MD, Department of Cardiovascular Surgery, Faculty of Medicine, Kyushu University, 3-1-1 Maidashi, Higashi-ku, Fukuoka 812-82, Japan.

Copyright (C) 1998 by Mosby, Inc.

$0022-5223 / 98 \$ 5.00+0 \quad \mathbf{1 2} / \mathbf{1} / \mathbf{8 9 2 9 2}$ 


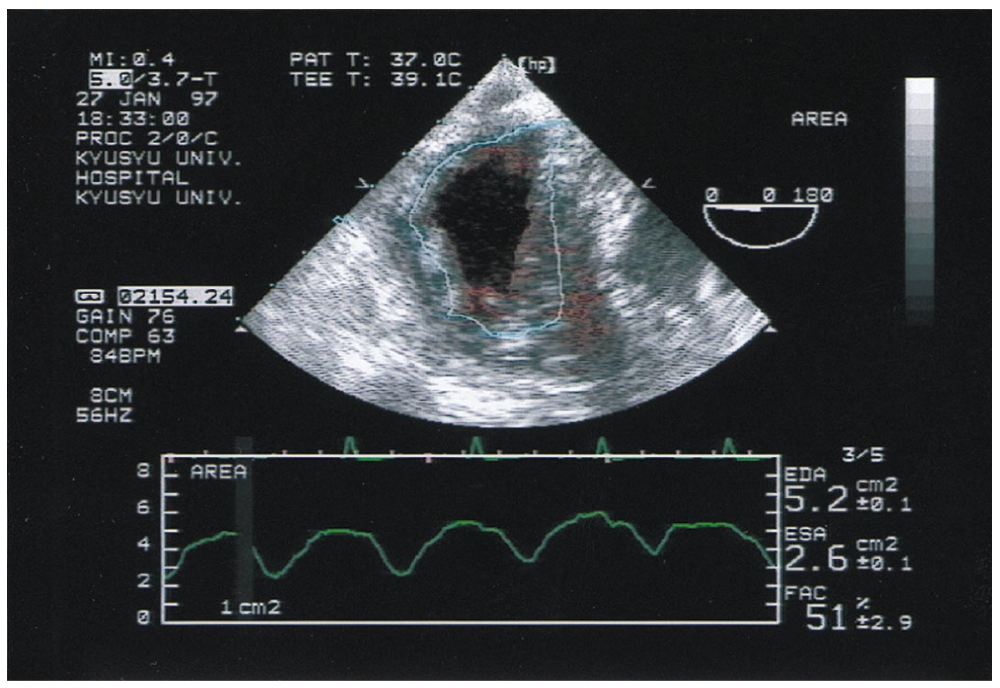

Fig. 1. An echocardiographic image in which the ABD outlines the blood-tissue interface, and the region of interest is circled in the right ventricular cavity. The right ventricular cross-sectional area signal appears at the bottom.

pressure, presumably as the result of reducing right ventricular afterload, but its effect of enhancement on right ventricular contractility has not been demonstrated. ${ }^{4}$ That may relate to the difficulties of assessing the load-independent parameters of right ventricular contractility. To use inotropic agents safely in patients with postoperative right ventricular dysfunction, it is essential to assess the relative contributions of positive inotropism and vasodilative actions of the agents.

We, therefore, applied the framework of pressure-volume relations and the concept of ventricular/arterial coupling to evaluate right ventricular performance. Ventricular/arterial coupling is a concept built on the framework of the ventricular pressure-volume relation. ${ }^{5}$ This concept enables one to discriminate between the positive inotropic and vasodilatory effect of the agents. Accordingly, the purpose of this study was to investigate the effects of amrinone on right ventricular contractility and right ventricular afterload in the patients who had undergone cardiac operation with a transesophageal echocardiography and catheter tip micromanometer to construct right ventricular pressure-cross-sectional area loops.

\section{Methods}

Patients. Studies were attemped in 13 patients who underwent elective cardiac operations. The study protocol was approved by the Human Subjects Committee of our institution. Informed consent was obtained from all the patients, who were hemodynamically stable in the intensive care unit at the time of study. Two patients were excluded because of poor quality echocardiographic images. The study group therefore consisted of 11 patients: 9 men and 2 women, aged $49 \pm 14$ years (range, 20 to 64 years). The operations performed were closure of atrial septal defect $(n=2)$, closure of atrial septal defect and mitral valve repair $(n=2)$, mitral valve replacement and tricuspid annuloplasty $(n=3)$, mitral valve repair and tricuspid annuloplasty $(n=2)$, mitral valve replacement and maze procedure $(n=1)$, and closure of atrial septal defect, mitral valve repair, and maze procedure $(n=1)$. None of the patients was in right ventricular failure or dysfunction evidenced by elevated right ventricular end-diastolic pressure.

Instrumentation. After surgery, patients were transferred to the intensive care unit and received controlled ventilation. The setting of the ventilator was unchanged (inspired oxygen fraction, 0.40 to 0.70 ; inspiration/expiration ratio, 1:2; positive end-expiratory pressure, 1 to $4 \mathrm{~mm}$ $\mathrm{Hg}$ ) throughout the protocol for each patient. We positioned a rapid-response thermistor pulmonary artery catheter (7.5F Swan-Ganz catheter, model 93A-431H; Baxter Healthcare Corp., Santa Ana, Calif.) in the pulmonary artery and a catheter-tip micromanometer (Sentron; AC Roden, The Netherlands) in the right ventricle. These two catheters were inserted percutaneously in the operating room after the induction of anesthesia. A $5 \mathrm{MHz}$ omniplane transesophageal echocardioscope (model HP 21362C; Hewlett-Packard, Andover, Mass.) was inserted to obtain a cross-sectional view of the right ventricle at the midpapillary muscle level of the left ventricle. Echocardiographic images were acquired with a Hewlett-Packard Sonos 2500 echocardiographic system (model M2406A; Hewlett-Packard) with automated border detection (ABD) capabilities. Images were recorded from the transgastric right ventricular mid-short-axis plane, at the left 


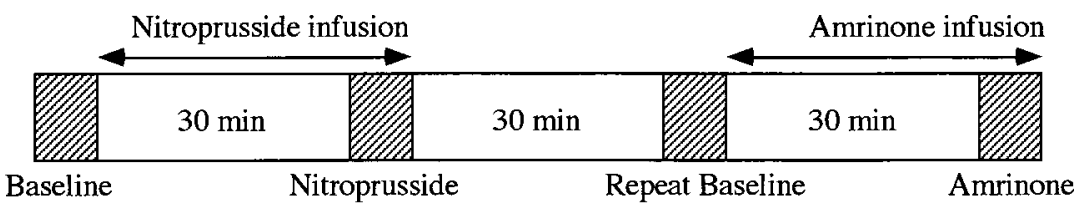

\section{Steady state pressure-area data} Multiple pressure-area loops by bolus nitroglycerin IV

Fig. 2. A time line of the protocol. The conventional hemodynamics data, steady-state beat right ventricular pressure-area data, and multiple pressure-area loops by the bolus nitroglycerin intravenously (IV) were measured at baseline, after nitroprusside infusion, at repeat baseline, and after amrinone infusion.

ventricular midpapillary muscle level as an anatomic landmark. A region of interest was then drawn manually immediately beyond the right ventricular endocardial border to exclude the left ventricular cavity and lowdensity ultrasound signals that may appear within the lateral myocardium (Fig. 1). Once the image had been established, the same region of interest and the gain setting were maintained throughout the protocol for each patient.

Protocol. The start of the protocol was about 5 hours after the removal of the aortic crossclamp. A time line of the protocol is given in Fig. 2. At first, the thermodilution cardiac output was measured, and the right ventricular pressure and cross-sectional area were recorded simultaneously to obtain steady-state beat data. Then a nitroglycerin $(0.05$ to $0.1 \mathrm{mg})$ bolus, intravenously, was made to reduce right ventricular peak pressure by $15 \%$ to obtain multiple pressure-area loops at different preload. These measurements were performed at baseline, after nitroprusside infusion, at repeat baseline, and after amrinone infusion (Fig. 2).

We administered nitroprusside as a pure dilator to compare the effects of amrinone. After baseline measurements, nitroprusside was infused intravenously at an initial rate of $0.3 \mu \mathrm{g} \cdot \mathrm{kg}^{-1} \cdot \mathrm{min}^{-1}$, with the dose raised in increments of $0.2 \mu \mathrm{g} \cdot \mathrm{kg}^{-1} \cdot \mathrm{min}^{-1}$ until the mean arterial pressure had fallen to $80 \%$ of baseline. Similar measurements were made 30 minutes after the start of infusion.

After discontinuation of nitroprusside, systemic arterial pressure, pulmonary artery pressure, and cardiac output were permitted to return to the baseline values. The repeat baseline hemodynamics and right ventricular pressure-area recordings were made. Amrinone was then infused intravenously at an initial bolus of $1 \mathrm{mg} \cdot \mathrm{kg}^{-1}$ over a 10 -minute period, followed by $10 \mu \mathrm{g} \cdot \mathrm{kg}^{-1} \cdot \mathrm{min}^{-1}$ drip infusion. After a wait of at least 30 minutes for the stabilization of pressures and cardiac output, final hemodynamic and right ventricular pressure-area recordings were made.

Other cardiotonic agents, such as dopamine (dose, 0 to $10 \mu \mathrm{g} \cdot \mathrm{kg}^{-1} \cdot \mathrm{min}^{-1}$; mean, $\left.2.9 \mu \mathrm{g} \cdot \mathrm{kg}^{-1} \cdot \mathrm{min}^{-1}\right)$ and dobutamine (dose, 0 to $8 \mu \mathrm{g} \cdot \mathrm{kg}^{-1} \cdot \min ^{-1}$; mean, $3.7 \mu \mathrm{g} \cdot \mathrm{kg}^{-}$ ${ }^{1} \cdot \min ^{-1}$ ) or vasodilating drugs (isosorbide dinitrate in a dose of 0 to $0.90 \mu \mathrm{g} \cdot \mathrm{kg}^{-1} \cdot \mathrm{min}^{-1}$; mean, 0.32 $\mu \mathrm{g} \cdot \mathrm{kg}^{-1} \cdot \mathrm{min}^{-1}$ ) were unchanged during the protocol for each patient.
Data acquisition and analysis. Right ventricular, left atrial, radial artery, pulmonary artery and central venous pressure, carbon dioxide concentration of expiratory gas waveform, and electrocardiographic (lead II) signals were monitored on a bedside monitor (M1166A; Hewlett-Packard, Inc., Sunnyvale, Calif.). All these signals and the analog right ventricular area signal from the echocardiography machine equipped with the $\mathrm{ABD}$ system were on-line, digitized at $200 \mathrm{~Hz}$ with an analog-to-digital converter (Mac Lab System; AD Instruments, Ltd., Dunedin North, New Zealand) and recorded on a laptop computer (Macintosh Power Book 550C; Apple Computer, Inc., Cupertino, Calif.). The right ventricular pressure and area signals were plotted to display pressure-area loops in real time. To synchronize right ventricular pressure data with the area data from the echocardiographic ABD system, the electrocardiography signals from both the patient monitors and the ABD system were recorded simultaneously. It has been known that there is a delay in right ventricular area signal. ${ }^{6}$ Our preliminary study directly comparing ventricular volume and area signal showed that the delay was $40 \mathrm{msec}$. Accordingly we advanced the right ventricular area signal by $40 \mathrm{msec}$ in all patients.

From the beats obtained at a steady state, maximal cross-sectional area $\left(\mathrm{A}_{\max }\right)$ and minimal cross-sectional area $\left(\mathrm{A}_{\min }\right)$ were obtained. Stroke area $\left(\mathrm{SA} ; \mathrm{SA}=\mathrm{A}_{\max }\right.$ $\left.-\mathrm{A}_{\text {min }}\right)$ and fractional area change $(\mathrm{FAC}$; $\mathrm{FAC}=$ $\left.\mathrm{SA} / \mathrm{A}_{\max }\right)$ were calculated. SA and FAC were used as the echocardiographic equivalents of stroke volume and ejection fraction, respectively. End-systolic elastance $\left(\mathrm{E}_{\mathrm{es}}\right)$ and the area axis intercept $\left(\mathrm{A}_{0}\right)$ of the end-systolic pressure-area relationship (ESPAR) were obtained in the following method: During the nitroglycerin bolus injection ( 0.05 to $0.1 \mathrm{mg}$ ), only the data during end-expiratory periods were selected for the analysis to minimize the effects of positive-pressure ventilation. For each beat during the nitroglycerin infusion, the point of maximal pressure/area was first determined. A least-squares linear regression was applied to generate slope $\left(\mathrm{E}_{\mathrm{es}}\right)$ and intercept $\left(A_{0}\right)$. With this $A_{0}$ estimate, the points of maximal $\left(\mathrm{P} /\left[\mathrm{A}-\mathrm{A}_{0}\right]\right)$ for each loop were recalculated, where $\mathrm{P}$ and A are right ventricular pressure and area, respectively, and a second regression was used to determine new estimates for $\mathrm{E}_{\mathrm{es}}$ and $\mathrm{A}_{0}$. This process was continued until the change between successive iterations for each parameter was less than 0.01 . Once $\mathrm{A}_{0}$ was obtained, the end- 
Table I. Effect of nitroprusside and amrinone on hemodynamic variables

\begin{tabular}{lcccc}
\hline & & & & \multicolumn{2}{c}{ Repeated measure } \\
of ANOVA
\end{tabular}

Exact $p$ values are described in the text. $A N O V A$, Analysis of variance; $M A P$, mean arterial pressure; $M P A P$, mean pulmonary arterial pressure; $L A P$, left atria pressure; $C V P$, central venous pressure; $C O$, cardiac output; $S V$, stroke volume; $S V R$, systemic vascular resistance; $P V R$, pulmonary vascular resistance; $R V E F$, right ventricular ejection fraction.

${ }^{*} p<0.05$ vs baseline.

$\dagger p<0.05$ vs repeat baseline.

systolic point $\left(\mathrm{A}_{\mathrm{es}}, \mathrm{P}_{\mathrm{es}}\right)$ of the steady-state beats was determined as the point that gave the maximal $\left(\mathrm{P} /\left[\mathrm{A}-\mathrm{A}_{0}\right]\right)$, where $\mathrm{A}_{\mathrm{es}}$ and $\mathrm{P}_{\mathrm{es}}$ are right ventricular end-systolic area and pressure, respectively. To determine the position of the ESPAR in the operating range, the area associated with the $P_{\mathrm{es}}$ of $25 \mathrm{~mm} \mathrm{Hg}$ was calculated as: $\mathrm{A}_{25}=\mathrm{A}_{0}+25 / \mathrm{E}_{\mathrm{es}}$.

The effective arterial elastance $\left(\mathrm{E}_{\mathrm{a}}\right)$ of the pulmonary artery was calculated as $\mathrm{P}_{\mathrm{es}} / \mathrm{SA}$. The coupling between the right ventricle and the pulmonary artery was determined by calculating $\mathrm{E}_{\mathrm{a}} / \mathrm{E}_{\mathrm{es}}$.

The total mechanical energy generated by ventricular contraction, defined by the pressure-volume area (PVA), was obtained as the area circumscribed by the end-systolic trajectory of the pressure-area loop, the end-systolic pressure-area line, and the end-diastolic pressure-area relationship. External work (EW) was calculated as the area inside the pressure-area loop. EW is equivalent to stroke work. The efficiency of energy transfer from PVA to EW in the right ventricle was evaluated as EW/PVA.

Statistics. Results are presented as mean \pm standard deviation. A repeated-measures of analysis of variance was used to determine the null hypothesis that the variables at the four points (baseline, nitroprusside, repeat baseline, and amrinone) were equal. If the null hypothesis was rejected by the $p$ value less than 0.05 , Bonferroni's adjustment was performed to examine the difference between the values of each intervention and its corresponding baseline values. Because $\mathrm{E}_{\mathrm{es}}$ and $\mathrm{A}_{0}$ are covariant via a linear regression equation, multiple linear regression analysis was performed by introducing two sets of dummy variables, one for the protocol (before and after drug infusion) and another for the patients. ${ }^{6}$ For all statistical analyses, SPSS (version 6.1J for the Macintosh; SPSS, Inc., Chicago, Ill.) was used.

\section{Results}

Effects of nitroprusside and amrinone on conventional hemodynamic variables. The effects of nitroprusside and amrinone on hemodynamic variables obtained from the thermodilution catheter are shown in Table I. With amrinone administration, the heart rate tended to be higher (from $90.6 \pm 5.8$ beats/min to $96.3 \pm 9.4$ beats/min), but neither drug significantly altered the heart rate. Both nitroprusside and amrinone significantly reduced mean arterial pressure and pulmonary artery pressure. Left atrial pressure decreased with amrinone (from $9.0 \pm$ $2.3 \mathrm{~mm} \mathrm{Hg}$ to $7.7 \pm 2.0 \mathrm{~mm} \mathrm{Hg}, p=0.038$ ) but not with nitroprusside (from $8.5 \pm 2.3 \mathrm{~mm} \mathrm{Hg}$ to $7.6 \pm$ $2.7 \mathrm{~mm} \mathrm{Hg}, p>0.2)$. Central venous pressure did not change significantly with either of the drugs. When nitroprusside was administered, there were no significant changes in cardiac output, stroke volume, and pulmonary vascular resistance, except for systemic vascular resistance (from $1525 \pm 683$ dyne $\cdot \mathrm{sec} \cdot \mathrm{cm}^{-5}$ to $1273 \pm 518$ dyne $\cdot \mathrm{sec} \cdot \mathrm{cm}^{-5}, p=$ 0.020 ). Amrinone did not cause the changes in systemic vascular resistance (from $1576 \pm 906$ dyne $\cdot \mathrm{sec} \cdot \mathrm{cm}^{-5}$ to $1043 \pm 379$ dyne $\cdot \mathrm{sec} \cdot \mathrm{cm}^{-5}, p=$ 0.060 ), cardiac output (from $4.80 \pm 2.27 \mathrm{~L} \cdot \mathrm{min}^{-1}$ to $\left.5.39 \pm 1.92 \mathrm{~L} \cdot \mathrm{min}^{-1}, p=0.122\right)$, stroke volume (from $52.5 \pm 23.4 \mathrm{ml}$ to $55.4 \pm 18.3 \mathrm{ml}$ ), and pulmonary vascular resistance (from $249 \pm 177$ dyne $\cdot \mathrm{sec} \cdot \mathrm{cm}^{-5}$ to $\left.185 \pm 122 \mathrm{dyne} \cdot \mathrm{sec} \cdot \mathrm{cm}^{-5}\right)$. Right ventricular ejection fraction from the rapid response thermodilution catheter did not show significant change with neither drug.

Effects of nitroprusside and amrinone on ventricular/arterial coupling and mechanical efficiency. Representative right ventricular pressure-area loops before and after nitroprusside and amrinone administration obtained by the bolus nitroglycerin intravenously are shown in Fig. 3. Multiple linear regres- 

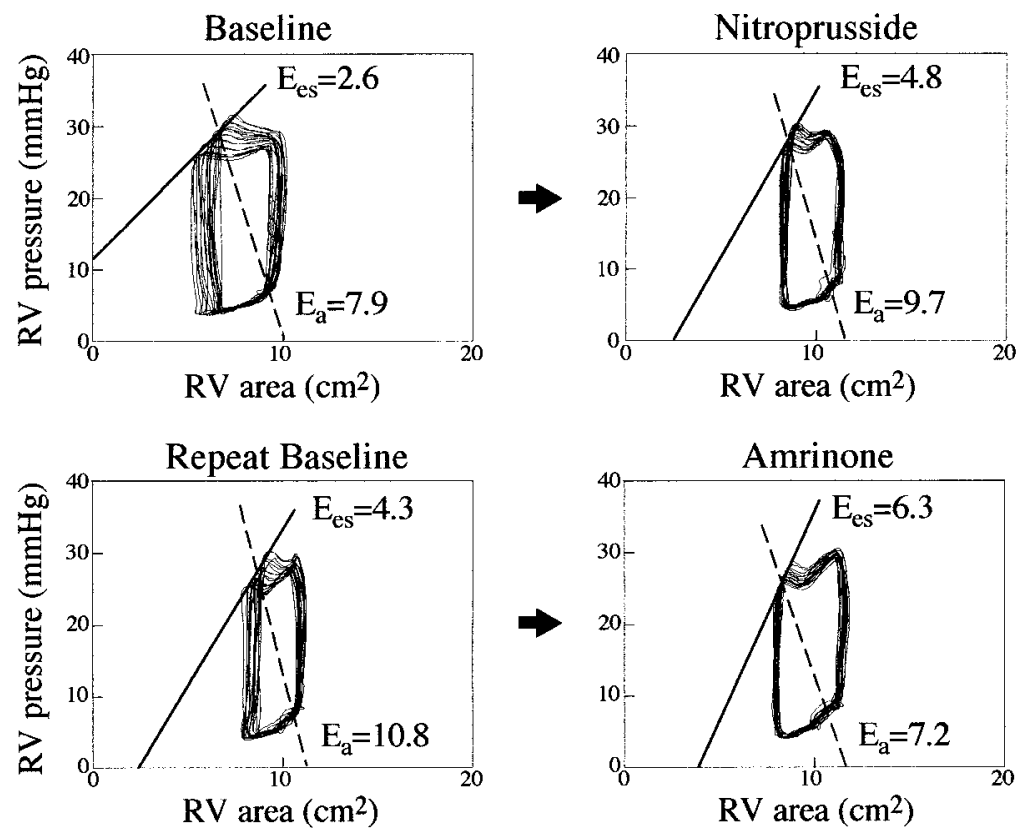

Fig. 3. Representative recordings of right ventricular $(R V)$ pressure-area loops before and after nitroprusside and amrinone administration obtained by the bolus nitroglycerin intravenously. Lines of the end-systolic pressure-area relation ( $\mathrm{E}_{\mathrm{es}}$; solid lines $)$ and lines of effective arterial elastance $\left(E_{a}\right.$; dotted lines) are shown. In this case, amrinone increased $\mathrm{E}_{\mathrm{es}}$ from 4.3 to $6.3 \mathrm{~mm} \mathrm{Hg} / \mathrm{cm}^{2}$, which implied the increase in right ventricular contractility. On the other hand, amrinone decreased $\mathrm{E}_{\mathrm{a}}$ from 10.8 to $7.2 \mathrm{~mm} \mathrm{Hg} / \mathrm{cm}^{2}$, which implied right ventricular afterload reduction.

sion analysis incorporating all data indicated that effects of amrinone were significant in altering $\mathrm{E}_{\mathrm{es}}$, the slope of the ESPAR $(p=0.007)$.

Fig. 4 shows the effects of nitroprusside and amrinone on variables defined by right ventricular pressure-area loops. A repeated-measures of analysis of variance revealed significant changes in $A_{o}$ $(p=0.029), \mathrm{E}_{\mathrm{a}}(p=0.002), \mathrm{E}_{\mathrm{a}} / \mathrm{E}_{\mathrm{es}}(p=0.012$,$) and$ EW/PVA $(p=0.015)$. As a group, amrinone increased $\mathrm{E}_{\mathrm{es}}\left(4.47 \pm 1.79 \mathrm{~mm} \mathrm{Hg} / \mathrm{cm}^{2}\right.$ to $6.56 \pm 2.22$ $\left.\mathrm{mm} \mathrm{Hg} / \mathrm{cm}^{2}, p=0.007\right)$, reduced $\mathrm{E}_{\mathrm{a}}(8.82 \pm 3.99$ $\mathrm{mm} \mathrm{Hg} / \mathrm{cm}^{2}$ to $\left.7.05 \pm 4.01 \mathrm{~mm} \mathrm{Hg} / \mathrm{cm}^{2}, p=0.004\right)$, and decreased the coupling ratio, $\mathrm{E}_{\mathrm{a}} / \mathrm{E}_{\mathrm{es}}(2.40 \pm$ 1.45 to $1.16 \pm 0.63, p=0.009)$, which resulted in the increase in EW/PVA $(0.44 \pm 0.15$ to $0.54 \pm 0.15$, $p=0.013)$. The increase in this ratio indicates improvement in the energy transfer from ventricle to arterial system. With amrinone, $\mathrm{A}_{0}$ significantly increased $\left(0.26 \pm 5.09 \mathrm{~cm}^{2}\right.$ to $3.22 \pm 4.25 \mathrm{~cm}^{2}, p=$ $0.048)$, but $\mathrm{A}_{25}$ remained unchanged (7.13 \pm 4.29 $\mathrm{cm}^{2}$ to $7.45 \pm 4.34 \mathrm{~cm}^{2}$ ).

The effects of nitroprusside were insignificant. The slight decrease in EW $(66.4 \pm 26.4 \mathrm{~mm}$ $\mathrm{Hg} \cdot \mathrm{cm}^{2}$ to $58.6 \pm 22.8 \mathrm{~mm} \mathrm{Hg} \cdot \mathrm{cm}^{2}$ ) and PVA $\left(152.5 \pm 73.5 \mathrm{~mm} \mathrm{Hg} \cdot \mathrm{cm}^{2}\right.$ to $134.4 \pm 66.2 \mathrm{~mm}$ $\mathrm{Hg} \cdot \mathrm{cm}^{2}$ ) did not reach statistical significance. Nitroprusside decreased mean aortic pressure more than $10 \%$ (from $80.8 \pm 4.5 \mathrm{~mm} \mathrm{Hg}$ to $69.3 \pm 6.3 \mathrm{~mm}$ $\mathrm{Hg}, p<0.001$ ), which implied that the dose of nitroprusside was large enough to influence systemic circulation.

Hemodynamic variables obtained from the baseline right ventricular pressure-area loops are shown in Table II. With nitroprusside, right ventricular peak-systolic pressure decreased by $8.5 \%$ (29.6 \pm $6.9 \mathrm{~mm} \mathrm{Hg}$ to $27.1 \pm 5.1 \mathrm{~mm} \mathrm{Hg}, p=0.043$ ), right ventricular end-systolic pressure decreased by $9.6 \%$ $(27.0 \pm 8.6 \mathrm{~mm} \mathrm{Hg}$ to $24.4 \pm 7.4 \mathrm{~mm} \mathrm{Hg}, p=0.046)$ and maximum right ventricular pressure development decreased by $7.8 \%(232.1 \pm 83.4 \mathrm{~mm} \mathrm{Hg} / \mathrm{sec}$ to $213.9 \pm 72.1 \mathrm{~mm} \mathrm{Hg} / \mathrm{sec}, p=0.025)$ with no change in right ventricular end-diastolic pressure, $\mathrm{A}_{\text {max }}, \mathrm{A}_{\text {min }}, \mathrm{SA}$, and FAC.

On the other hand, amrinone decreased right ventricular peak-systolic pressure in 9 of 11 patients although there was no significant difference $(30.0 \pm$ $5.0 \mathrm{~mm} \mathrm{Hg}$ to $28.4 \pm 6.3 \mathrm{~mm} \mathrm{Hg}, p=0.162$ ). Right ventricular end-systolic pressure decreased by 


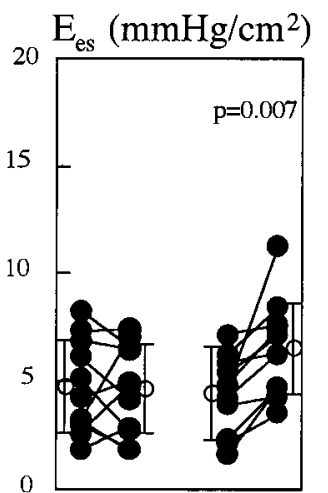

Nitroprusside Amrinone

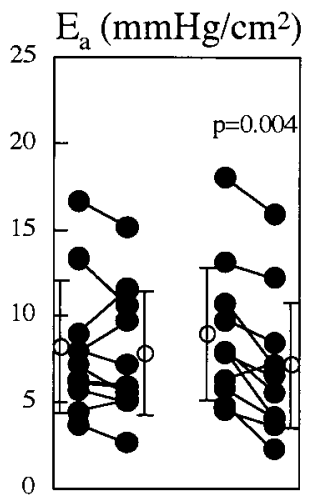

Nitroprusside Amrinone
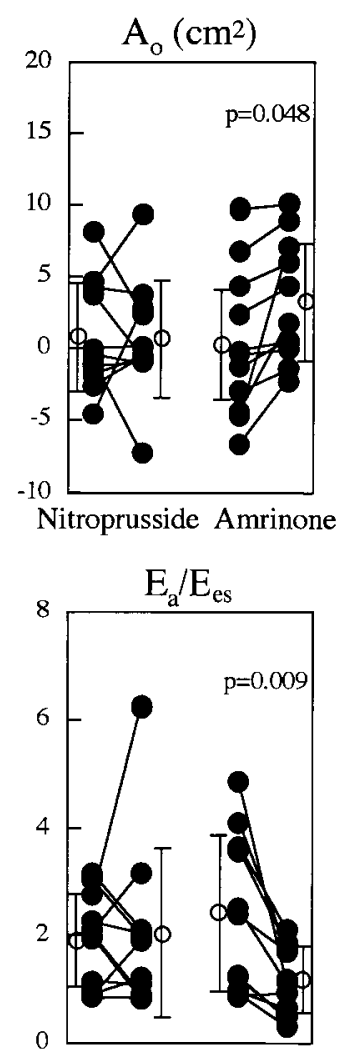

Nitroprusside Amrinone
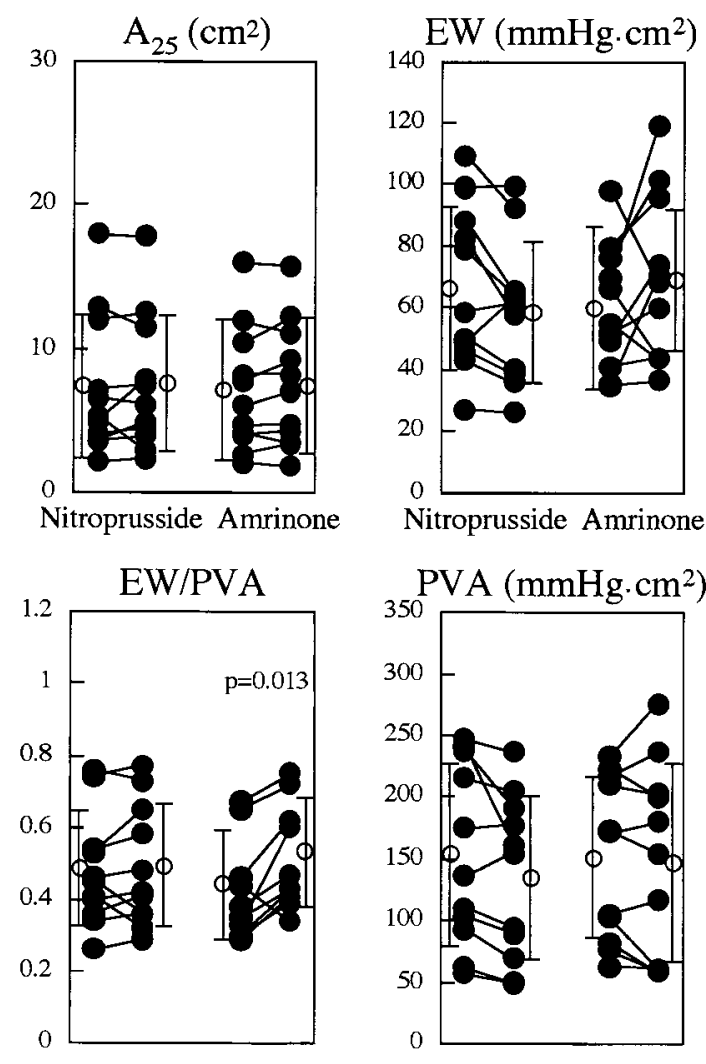

Nitroprusside Amrinone
Nitroprusside Amrinone

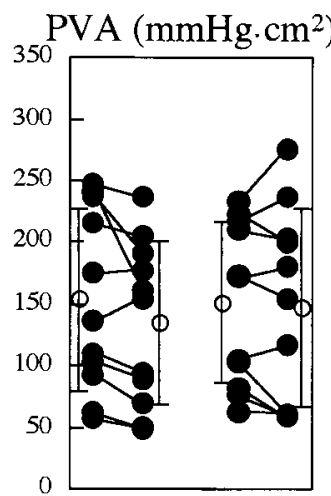

Nitroprusside Amrinone

Fig. 4. Changes in $E_{e s}$, the intercept of $\operatorname{ESPAR}\left(\mathrm{A}_{\mathrm{o}}\right)$, the area associated with the $\mathrm{P}_{\mathrm{es}}$ of $25 \mathrm{~mm} \mathrm{Hg}\left(\mathrm{A}_{25}\right)$, $E W$, effective $E_{a}$, the ratio of $E_{a}$ to $E_{e s}\left(E_{a} / E_{e s}\right)$, PVA and the efficiency of energy transfer from PVA to EW (EW/PVA) after administration of nitroprusside and amrinone in the same patients with paired data sets. Mean $\pm \mathrm{SD}$ values are also shown.

Table II. Hemodynamic variables obtained from the baseline $R V$ pressure-area loops

\begin{tabular}{|c|c|c|c|c|c|}
\hline & Baseline & Nitroprusside & Repeat baseline & Amrinone & $\begin{array}{c}\text { Repeated-measures } \\
\text { of } A N O V A\end{array}$ \\
\hline $\mathrm{RVP}_{\text {PEAK }}(\mathrm{mm} \mathrm{Hg})$ & $29.6 \pm 6.9$ & $27.1 \pm 5.1^{*}$ & $30.0 \pm 5.0$ & $28.4 \pm 6.3$ & $p=0.005$ \\
\hline $\mathrm{RVP}_{\mathrm{es}}(\mathrm{mm} \mathrm{Hg})$ & $27.0 \pm 8.6$ & $24.4 \pm 7.4^{*}$ & $27.8 \pm 6.3$ & $23.1 \pm 82 \dagger$ & $p<0.001$ \\
\hline $\mathrm{RV} \mathrm{dp} / \mathrm{dt}_{\max }(\mathrm{mm} \mathrm{Hg} / \mathrm{sec})$ & $232.1 \pm 83.4$ & $213.9 \pm 72.1^{*}$ & $239.6 \pm 80.4$ & $246.2 \pm 88.5$ & $p<0.001$ \\
\hline RVEDP $(\mathrm{mm} \mathrm{Hg})$ & $5.9 \pm 1.4$ & $5.6 \pm 1.8$ & $6.5 \pm 1.8$ & $5.7 \pm 1.9 \dagger$ & $p=0.161$ \\
\hline $\mathrm{A}_{\max }\left(\mathrm{cm}^{2}\right)$ & $10.0 \pm 4.8$ & $9.7 \pm 5.0$ & $10.2 \pm 4.8$ & $10.0 \pm 5.3$ & $p=0.530$ \\
\hline$A_{\min }\left(\mathrm{cm}^{2}\right)$ & $6.4 \pm 3.9$ & $6.2 \pm 4.3$ & $6.7 \pm 4.2$ & $6.4 \pm 6.3$ & $p=0.414$ \\
\hline $\mathrm{SA}\left(\mathrm{cm}^{2}\right)$ & $3.7 \pm 1.2$ & $3.5 \pm 1.1$ & $3.5 \pm 1.1$ & $3.7 \pm 1.2$ & $p=0.653$ \\
\hline FAC $(\%)$ & $40.6 \pm 12.1$ & $42.4 \pm 17.2$ & $38.5 \pm 13.4$ & $42.4 \pm 13.2$ & $p=0.314$ \\
\hline
\end{tabular}

Exact $p$ values are described in the text. $A N O V A$, Analysis of variance; $R V P_{P E A K}$, right ventricular peak-systolic pressure; $R V P_{e s}$, right ventricular end-systolic pressure; $R V d p / d t_{\max }$, maximum right ventricular pressure development; $R V E D P$, right ventricular end-diastolic pressure.

${ }^{*} p<0.05$ vs baseline.

$\dagger p<0.05$ vs repeat baseline.

$16.9 \%(27.8 \pm 6.3 \mathrm{~mm} \mathrm{Hg}$ to $23.1 \pm 8.2 \mathrm{~mm} \mathrm{Hg}, p=$ 0.006 ), but maximum right ventricular pressure development remained unchanged $(239.6 \pm 80.4 \mathrm{~mm}$ $\mathrm{Hg} / \mathrm{sec}$ to $246.2 \pm 88.5 \mathrm{~mm} \mathrm{Hg} / \mathrm{sec}$ ) with no change in right ventricular end-diastolic pressure $(6.5 \pm 1.8$ $\mathrm{mm} \mathrm{Hg}$ to $5.7 \pm 1.9 \mathrm{~mm} \mathrm{Hg}$ ). There was no significant change in $\mathrm{A}_{\max }, \mathrm{A}_{\min }$, and SA. FAC measured by echocardiography increased in 10 of 11 
patients with amrinone, but this difference was not significant $(38.5 \% \pm 13.4 \%$ to $42.4 \% \pm 13.2 \%)$.

\section{Discussion}

We investigated the effects of amrinone on right ventricular contractility and right ventricular afterload using the concept of ventricular/arterial coupling. Amrinone reduced afterload (decrease in $E_{a}$ ) and enhanced contractility (increase in $\mathrm{E}_{\mathrm{es}}$ ). Accordingly, there was an improvement in the mechanical efficiency (increased EW/PVA) because of both afterload reduction and contractility enhancement.

The conventional measures of ventricular function, such as ejection fraction or cardiac output, are afterload dependent. Thus it has been difficult to assess inotropic action of the agents such as amrinone, which also possesses vasodilatory effect. The framework of pressure-volume relationship and the concept of ventricular/arterial coupling made it possible to separately assess the inotropic and afterload reducing effects of the agents. This approach has been applied to study the effects of pharmacologic agents with inotropic and vasodilatory effects in the human left ventricle. ${ }^{7-10}$ Regarding amrinone, Kass and colleagues ${ }^{11}$ succeeded in assessing the effects of amrinone on left ventricular contractility and afterload using the framework of left ventricular pressure-volume relationship in the intact canine left ventricle. In the right ventricle, however, the number of studies has been limited because of the difficulty in measuring right ventricular volume. Using radionuclide ventriculography, Konstam and colleagues $^{4}$ studied the effect of amrinone on right ventricular systolic function. They compared amrinone and nitroprusside by measuring pulmonary artery end-systolic pressure and right ventricular end-systolic volume. They intended to demonstrate a larger decrease in right ventricular end-systolic volume with amrinone than with nitroprusside at a similar systolic pressure. But the difference in right ventricular end-systolic volume did not reach statistical significance, probably because of the reduced left ventricular-to-right ventricular interdependence, which will be discussed later. In addition, they did not measure right ventricular $\mathrm{E}_{\mathrm{es}}$ directly during amrinone infusion. To the best of our knowledge, our study is the first to demonstrate the inotropic effect of amrinone on the right ventricle by directly elucidating the increase in right ventricular $\mathrm{E}_{\mathrm{es}}$.

The assessment of right ventricular $\mathrm{E}_{\mathrm{es}}$ was made possible with the technology of an on-line, real-time measurement of right ventricular cross-sectional area as a surrogate for right ventricular volume. ${ }^{12,13}$ This technology, ABD, was crucial for investigating right ventricular pressure-volume relationship, because instantaneous measurement of right ventricular pressure and area greatly improved the quality of study. The standard method of studying the left ventricular pressure-volume relationship is by use of a conductance catheter for volume measurement. ${ }^{7-11,14}$ In the right ventricle, however, complex geometry of the right ventricle has hindered the on-line determination of volume in situ. Accordingly, pressure-volume assessment of the right ventricle has been difficult. ${ }^{15-19}$ With the ABD technology, real-time display of the right ventricular pressure-area loops became possible at the bedside of the patient in the intensive care unit.

Feasibility of using the right ventricular crosssectional area as a surrogate for right ventricular volume should be discussed briefly. Morita and associates ${ }^{20}$ were the first to use the right ventricular cross-sectional area to construct human right ventricular pressure-area loops in an intraoperative cardiac study. They manually traced the endocardial border of the right ventricle to measure the right ventricular cross-sectional area. The ABD was first applied to the human right ventricle by Gorcsan and his colleagues, ${ }^{12}$ who demonstrated the inotropic effect of dobutamine on the right ventricle in patients with end-stage heart failure. The validation of this approach was confirmed by Oe and colleagues, ${ }^{13}$ who used isolated canine heart preparation that contracted isovolumically. They studied the relation between right ventricular cross-sectional area obtained by echocardiographic $\mathrm{ABD}$ and true right ventricular volume directly measured by a balloon placed in the right ventricle with the left ventricular and right ventricular volumes controlled independently. They showed a highly linear correlation between the right ventricular area and volume, demonstrating the validity of the use of area as a surrogate for volume. We performed a preliminary intraoperative study that measured volumetric pulmonary artery flow by an electromagnetic flow probe and right ventricular cross-sectional area with ABD. There was a close relationship between the flow probe-measured stroke volume and SA (the difference between maximum and minimum crosssectional area), which indicated that ABD technology may be used to study human right ventricular function.

In our present study, there was an increase in $\mathrm{A}_{0}$ during amrinone infusion. In our preliminary study 
with dobutamine infusion, ${ }^{21}$ we observed classic changes in right ventricular ESPAR, that is, an increase in $\mathrm{E}_{\mathrm{es}}$ with no change in $\mathrm{A}_{0}$. These differences between dobutamine and amrinone administration could be related to the cross-talk from the left ventricle to the right ventricle. Elzinga and colleagues $^{22}$ found that when the left ventricle was decompressed, right ventricular pump function was impaired. Yamaguchi and colleagues ${ }^{23}$ showed, in an isovolumically contracting isolated canine heart preparation, that when left ventricular volume was decreased from $20 \mathrm{ml}$ to $10 \mathrm{ml}, \mathrm{E}_{\mathrm{es}}$ decreased by $9 \%$, and the volume axis intercept of the end-systolic pressure-volume relation shifted to the right. Morita and colleagues ${ }^{20}$ examined the right ventricular ESPAR before and after a left ventricular assist device implantation. After the unloading of the left ventricle by a left ventricular assist device, the right ventricular ESPAR significantly shifted to the right (increase in $\mathrm{A}_{0}$ ). Moon and associates ${ }^{24}$ explained the mechanism of decreased right ventricular pump performance by the reduced stretch of the ventricular septum, which caused the reduction of the Frank-Starling mechanism to work in the ventricular septum. In our preliminary study with dobutamine infusion, ${ }^{21}$ there was no change in left atrial pressure, whereas left atrial pressure decreased by $14 \%$ with amrinone in our current study. In addition, there was no significant decrease in left atrial pressure with nitroprusside. Therefore the shift of the right ventricular ESPAR to the right with amrinone could be the result of the reduced left ventricular volume, provided that there was no alteration in the left ventricular end-diastolic volume to left atrial pressure relationship. Further study is warranted to directly elucidate the mechanism.

The effects of amrinone on right ventricular afterload (decrease in $\mathrm{E}_{\mathrm{a}}$ ) and on contractility (increase in $E_{\text {es }}$ ) worked favorably to improve the mechanical efficiency of the right ventricle (EW/ PVA). The concept of ventricular/arterial coupling predicts that mechanical efficiency is given by the formula: $\mathrm{EW} / \mathrm{PVA}=1 /\left(1+0.5 \mathrm{E}_{\mathrm{a}} / \mathrm{E}_{\mathrm{es}}\right){ }^{25}$ In accordance with this prediction, $47 \%$ increase in $\mathrm{E}_{\mathrm{es}}$ and $20 \%$ decrease in $\mathrm{E}_{\mathrm{a}}$ resulted in $23 \%$ increase in EW/PVA. The improvement of EW/PVA was largely due to the increase in EW, whereas nitroprusside reduced both EW (12\%) and PVA (12\%), which failed to improve EW/PVA. The dosage of nitroprusside was sufficient to reduce systemic and pulmonary pressure, which could reflect venodilator effects that lowered pressures throughout the circu- lation without any direct reduction in pulmonary vascular resistance or $\mathrm{E}_{\mathrm{a}}$. Amrinone is thus preferred to nitroprusside for the purpose of effectively reducing right ventricular afterload.

The ABD algorithm analyzes backscatter data, which are affected by ultrasound gain settings. ${ }^{26}$ Because these gain settings involve manual adjustment based on visual inspection, complete objectivity of settings cannot be ensured; this is a limitation of this technique. Moreover, the method of measuring transesophageal echocardiographic area is dependent on the direction and placement of the echocardiography probe. Large tidal volume ventilation may alter the relation of the heart to the ultrasound sector and thus move the right ventricular cavity outside of the regionally adjusted gain settings. We thus selected only end-expiratory beats for the analyses to minimize the effects of positivepressure ventilation. In addition, the operator of the echocardiographic system was fixed to one person (Y.O.) throughout this study.

In conclusion, amrinone increased right ventricular contractility, reduced afterload, and improved the right ventricular mechanical efficiency. Using right ventricular pressure-area relations obtained with transesophageal echocardiography, we could successfully separate the simultaneous change in right ventricular systolic mechanics and afterload caused by amrinone. This methods may be a promising tool to quantify the relative vasodilating versus inotropic influences of pharmacologic agents in the right ventricle and to investigate which drug is most suitable to treat right ventricular heart failure and pulmonary hypertension. Amrinone administration may be beneficial in cardiac surgical patients with right ventricular dysfunction secondary to postoperative pulmonary hypertension and with primary right ventricular failure.

This study was prepared in consultation with Yasuhiko Harasawa, MD, for statistical analyses.

\section{REFERENCES}

1. Colucci WS, Wright RF, Braunwald E. New positive inotropic agents in the treatment of congestive heart failure: mechanisms of action and recent clinical developments. N Engl J Med 1986;314:349-58.

2. Konstam MA, Cohen SR, Weiland DS, Martin TT, Das D, Isner JM, et al. Relative contributions of inotropic and vasodilator effects to amrinone-induced hemodynamic improvement in congestive heart failure. Am J Cardiol 1986;57: 242-8.

3. Reddy VM, Hendricks-Munoz KD, Rajasinghe HA, Petrossian E, Hanley FL, Fineman JR. Post-cardiopulmonary by- 
pass pulmonary hypertension in lambs with increased pulmonary blood flow: a role for endothelin 1. Circulation 1997;95: 1054-61.

4. Konstam MA, Cohen SR, Salem DN, Das D, Aronovitz MJ, Brockway BA. Effect of amrinone on right ventricular function: predominance of afterload reduction. Circulation 1986; 74:359-66

5. Sunagawa K, Maughan WL, Burkhoff D, Sagawa K. Left ventricular interaction with arterial load studies in isolated canine ventricle. Am J Physiol 1983;245:H773-80.

6. Glanz SA, Slinker BK. Repeated measures. In: Glanz SA, editor. Primer of applied regression and analysis of variance. New York: McGraw-Hill; 1990. p.381-404.

7. Gorcsan J III, Gasior TA, Mandarino WA, Deneault LG, Hattler BG, Pinsky MR. Assessment of the immediate effects of cardiopulmonary bypass on left ventricular performance by on-line pressure-area relations. Circulation 1994;89:180-90

8. Takaoka H, Takeuchi M, Odake M, Hayashi Y, Mori M, Hata K, et al. Comparison of the effects on arterial-ventricular coupling between phosphodiesterase inhibitor and dobutamine in the diseased human heart. J Am Coll Cardiol 1993;22:598-606.

9. Feldman MD, Pak PH, Wu CC, Haber HL, Heesch CM, Bergin JD, et al. Acute cardiovascular effects of OPC-18790 in patients with congestive heart failure: time- and dosedependence analysis based on pressure-volume relations. Circulation 1996;93:474-83.

10. Mori M, Takeuchi M, Takaoka H, Hata K, Hayashi Y, Yokoyama M. Effect of NKH477, a new water-soluble forskolin derivative, on arterial-ventricular coupling and mechanical energy transduction in patients with left ventricular systolic dysfunction: comparison with dobutamine. J Cardiovasc Pharmacol 1994;24:310-6.

11. Kass DA, Grayson R, Marino P. Pressure-volume analysis as a method for quantifying simultaneous drug (amrinone) effects on arterial load and contractile state in vivo. J Am Coll Cardiol 1990;16:726-32.

12. Gorcsan J III, Murali S, Counihan PJ, Mandarino WA, Kormos RL. Right ventricular performance and contractile reserve in patients with severe heart failure. Circulation 1996;94:3190-7.

13. Oe M, Gorcsan J III, Mandarino WA, Kawai A, Griffith BP, Kormos RL. Automated echocardiographic measures of right ventricular area as an index of volume and end-systolic pressure-area relations to assess right ventricular function. Circulation 1995;92:1026-33.

14. Baan J, van der Velde ET, de Bruin HG, Smeenk GJ, Koops $\mathrm{J}$, van Dijk $\mathrm{AD}$, et al. Continuous measurement of left ventricular volume in animals and humans by conductance catheter. Circulation 1984;70:812-23.

15. Brown KA, Ditchey RV. Human right ventricular endsystolic pressure-volume relation defined by maximal elastance. Circulation 1988;78:81-91.

16. Dell'Italia LJ, Walsh RA. Application of a time varing elastance model to right ventricular performance in man. Cardiovasc Res 1988;22:864-74.

17. Redington AN, Gray HH, Hodson ME, Rigby ML, Oldershaw PJ. Characterization of the normal right ventricular pressure-volume relation by biplane angiography and simultaneous micromanometer pressure measurements. $\mathrm{Br}$ Heart J 1988;59:23-30.

18. Feneley MP, Elbeery JR, Gaynor JW, Gall SA, Davis JW, Rankin JS. Ellipsoidal shell subtraction model of right ventricular volume: comparison with regional free wall dimensions as indexes of right ventricular function. Circ Res 1990;67:1427-36.

19. Oldershaw P, Bishop A. The difficulties of assessing right ventricular function. Br Heart J 1995;74:99-100.

20. Morita S, Kormos RL, Mandarino WA, Eishi K, Kawai A, Gaisior TA, et al. Right ventricular/arterial coupling in the patient with left ventricular assistance. Circulation 1992; 86(Suppl):II316-25.

21. Ochiai Y, Morita S, Tanoue Y, Tominaga R, Yasui $\mathrm{H}$. On-line evaluation of load independent right ventricular function using transesophageal echo with automated border detection [Abstract]. Circulation 1996;94(Suppl):I379.

22. Elzinga G, Piene H, de Jong JP. Left and right ventricular pump function and consequences of having two pump in one heart: a study on the isolated cat heart. Circ Res 1980;45:564-74.

23. Yamaguchi S, Tsuiki K, Miyawaki H, Tamada Y, Ohta I, Sukekawa H, et al. Effect of left ventricular volume on right ventricular end-systolic pressure-volume relation: resetting of regional preload in right ventricular free wall. Circ Res 1989;65:623-31.

24. Moon MR, Bolger AF, DeAnda A, Komeda M, Daughers II GT, Nikolic SD, et al. Septal function during left ventricular unloading. Circulation 1997;95:1320-7.

25. Nozawa T, Yasumura Y, Futaki S, Tanaka N, Uenishi M, Suga H. Efficiency of energy transfer from pressurevolume area to external mechanical work increases with contractile state and decreases with afterload in the left ventricle of the anesthetized closed-chest dog. Circulation 1988;77:1116-24

26. Bednarz JE, Marcus RH, Lang RM. Technical guidelines for performing automated border detection studies. J Am Soc Echocardiogr 1995;8:293-305. 